\title{
BOUNDARY VALUE PROBLEMS FOR SECOND ORDER NONLINEAR MATRIX DIFFERENTIAL EQUATIONS
}

\author{
WARREN E. SHREVE
}

\begin{abstract}
Existence of a solution to $X^{\prime \prime}=F\left(t, X, X^{\prime}\right), X(a)=A, X(b)=B$, is shown under certain conditions using a degree-theoretic result similar to one of Bebernes where $X, F, A, B$ are $n \times n$ matrices.
\end{abstract}

The paper [11] of Tomastik is concerned with oscillation of nonlinear matrix differential equations. In the present paper the concern is with the solution of boundary value problems for such equations. In fact it is shown here that a result similar to that of Bebernes [1] may be applied to obtain the existence of solutions of boundary value problems.

In particular, it is proven that under certain conditions including $A, B$ and $F$ positive definite $n \times n$ matrices, the boundary value problem

$$
\begin{gathered}
X^{\prime \prime}=F(t, X), \\
X(a)=A, \quad X(b)=B
\end{gathered}
$$

has a positive semidefinite solution.

Also an existence result is obtained for the more general matrix differential equation

$$
X^{\prime \prime}=F\left(t, X, X^{\prime}\right)
$$

with the same boundary conditions where specific bounds are imposed on $F$.

These problems have been studied previously by the author but little progress was made until the present methods based on degree theory were considered. For the interested reader a presentation of degree theory may be found in Schwartz [10].

Let $I=[a, b]$, and $R^{m}$, with $m=n^{2}$, be the space of $n \times n$ matrices with Euclidean operator norm. Let $D \subseteq I \times R^{m} \equiv S$ and $D^{\prime} \subseteq I \times R^{m} \times R^{m} \equiv S^{\prime}$ be bounded open sets in the relative topologies of $S$ and $S^{\prime}$, respectively, such that $T \equiv\{(t, Q(t)): t \in I\} \subseteq D$ and $T^{\prime} \equiv\{(t, Q(t),(B-A) /(b-a)): t \in I\} \subseteq D^{\prime}$, where $Q(t)=(b-t)(b-a)^{-1} A+(t-a)(b-a)^{-1} B$ is the linear matrix function connnecting $(a, A)$ and $(b, B)$. Let $\bar{U}$ denote the closure of $U$ in the appropriate topology.

Received by the editors April 14, 1977 and, in revised form, April 4, 1980. Presented at the Annual Meeting of the Society in San Antonio, Texas, January 22, 1976 as a preliminary report.

1980 Mathematics Subject Classification. Primary 34B15; Secondary 34A40.

Key words and phrases. Nonlinear matrix differential equations, boundary value problems, degree theory.

(C) 1981 American Mathematical Society 0002-9939/81/0000-0414/\$02.50 
Two standard results which are related to the questions considered in this note are found in Hartman [5, Corollary 4.2, p. 425] and Jackson [8, Theorem 2.1, p. 309]. The latter was originally published in Fountain and Jackson [4] and is valid for more general boundary value problems in the scalar case. The former result is valid for vector differential equations. The following theorem is closely related to both.

Theorem. Assume $f: I \times R^{n} \times R^{n} \rightarrow R^{n}$ is continuous. Let $M>0, N>0$ be given real numbers and let $q$ be the maximum of $\left\|f\left(t, x, x^{\prime}\right)\right\|$ on the compact set $\left\{\left(t, x, x^{\prime}\right): a \leqslant t \leqslant b,\|x\| \leqslant 2 M,\left\|x^{\prime}\right\| \leqslant 2 N\right\}$. Then, if

$$
\delta=\min \left[(8 M / q)^{1 / 2}, 2 N / q\right] \text {, }
$$

any boundary value problem $x^{\prime \prime}=f\left(t, x, x^{\prime}\right), x\left(t_{1}\right)=x_{1}, x\left(t_{2}\right)=x_{2}$, such that $\left[t_{1}, t_{2}\right]$ $\subseteq I, t_{2}-t_{1}<\delta,\left\|x_{1}\right\| \leqslant M,\left\|x_{2}\right\| \leqslant M$, and $\left\|\left(x_{2}-x_{1}\right) /\left(t_{2}-t_{1}\right)\right\|<N$, has $a$ vector solution $x$ such that $x^{\prime \prime}$ is continuous on $\left[t_{1}, t_{2}\right]$.

The proof is similar to that of [8, pp. 309-310] with allowances made for the fact that $f$ is a vector, including the use of the euclidean vector norm of $f$ in place of the absolute value of $f$.

Corollary. If $f: I \times R^{n} \times R^{n} \rightarrow R^{n}$ is bounded and continuous, then all boundary value problems $x^{\prime \prime}=f\left(t, x, x^{\prime}\right), x(a)=x_{a}, x(b)=x_{b}$ have solutions.

This corollary shows that the existence proved below in Theorem 3 was previously known. However, the theorem sheds light on the nature of solutions.

Two-point boundary value problems have been studied in a Banach space setting by Heimes [6], [7] where several interesting theorems are proven for equations of the form

$$
y^{\prime \prime}=A(t) y^{\prime}+B(t) y+f(t)
$$

where $A(t)$ and $B(t)$ operate on the elements of the Banach space.

For $U$ and $V$ square matrices, $U>V$ or $V<U$ and $U>V$ or $V<U$ will mean that $U$ and $V$ are symmetric and $U-V$ is positive definite and positive semidefinite, respectively. The identity and zero matrices will be denoted by $E$ and 0 , respectively. For every matrix $X$ satisfying $X \geqslant 0$ there is a unique matrix $Y$ denoted by $\sqrt{X}$ such that $Y \geqslant 0$ and $Y^{2}=X$. See Bellman [3, p. 43]. Further, define $|X|=\sqrt{X^{*} X}$. In this paper '*' denotes transpose.

Consider the following matrix differential equations:

$$
\begin{aligned}
& X^{\prime \prime}=\lambda F(t, X), \\
& X^{\prime \prime}=\lambda F\left(t, X, X^{\prime}\right),
\end{aligned}
$$

where $\lambda \in[0,1]$ and $F$ is assumed to be continuous.

Similar to condition $(\mathrm{H})$ of Bebernes [1] are

(H) If $X(t)$ is a solution of $\left(3_{\lambda}\right)-(2)$ then $(t, X(t)) \in D$ for all $t \in I$ or there exists $\tau \in I$ such that $(\tau, X(\tau)) \notin \bar{D}$.

$\left(\mathrm{H}^{\prime}\right)$ If $X(t)$ is a solution of $\left(3_{\lambda}^{\prime}\right)-(2)$ then $\left(t, X(t), X^{\prime}(t)\right) \in D^{\prime}$ for all $t \in I$ or there exists $\tau \in I$ such that $\left(\tau, X(\tau), X^{\prime}(\tau)\right) \notin \overline{D^{\prime}}$. 
TheOrem 1 (SEe Bebernes [1, TheOREM 2.1]). The boundary value problem (1)-(2), has a solution residing in $D$ if $(\mathrm{H})$ holds. Likewise $\left(1^{\prime}\right)-(2)$ has a solution in $D^{\prime}$ if $\left(\mathrm{H}^{\prime}\right)$ holds.

Proof. Consider ( $\left.1^{\prime}\right)$-(2). A parallel proof is valid for (1)-(2). Define $\Sigma^{\prime}=\{X \in$ $\left.C^{\prime}[a, b]: X(a)=A, X(b)=B\right\}$, and $\Omega^{\prime}=\left\{X \in \Sigma^{\prime}:\left(t, X(t), X^{\prime}(t)\right) \in D^{\prime}, t \in[a\right.$, $b$ ] . Let $G(t, s)$ be the Green's function for the boundary value problem

$$
X^{\prime \prime}=0, \quad X(a)=X(b)=0 .
$$

With $Q(t)$ as above, define the operator $T$ as follows:

$$
(T X)(t)=\int_{a}^{b} G(t, s) F\left(s, X(s), X^{\prime}(s)\right) d s+Q(t) .
$$

It can be shown that $T: \overline{\Omega^{\prime}} \rightarrow \Sigma^{\prime}, T$ is continuous and $\operatorname{cl}\left(T\left(\overline{\Omega^{\prime}}\right)\right)$ is compact in $\Sigma^{\prime}$. Thus we may consider the degree of $T$. Now $\operatorname{deg}\left(E, \Omega^{\prime}, Q\right)=1$ where $E$ is the identity and $Q \in \Omega^{\prime}$. However, $Q \notin(E-\lambda T)\left(\partial \Omega^{\prime}\right)$ for any $\lambda \in[0,1]$. For if $(E-\lambda T) X=Q$ for some $X \in \partial \Omega^{\prime}$, then $X$ would be a solution of $\left(3{ }_{\lambda}^{\prime}\right)$ which violates $\left(H^{\prime}\right)$. Thus, by the invariance under compact homotopy property of Leray-Schauder degree [10, p. 92], $\operatorname{deg}\left(E-T, \Omega^{\prime}, Q\right)=1$. Hence, by the existence property [10, p. 88], there is an $X \in \Omega^{\prime}$ such that $(E-T) X=Q$. Thus, one has a solution to $\left(1^{\prime}\right)-(2)$.

THEOREM 2. For $X$ an $n \times n$ matrix let $F(t, X)$ be a continuous $n \times n$ symmetric matrix depending on $t \in I$ and on each element $x_{i j}$ of the matrix $X$ such that $F(t, X)$ is positive (negative) semidefinite if any eigenvalue of $X$ is positive (negative). Let $A$ and $B$ be symmetric $n \times n$ matrices. Then the boundary value problem (1)-(2) has $a$ symmetric solution. In addition, if $A$ and $B$ are both positive definite then the solution is positive semidefinite for all $t \in I$. Furthermore, $A$ and $B$ both negative definite implies that $X(t)$ is negative semidefinite.

Note, for Theorem 2, a matrix function $U(t)$, with $U(t)>0$ and $U^{\prime \prime}(t)<0$, is an upper solution of (1) in the sense that $U^{\prime \prime}(t) \leqslant 0<F(t, U(t))$. Similarly, there are lower solutions. In particular, constant positive (negative) definite matrix functions are upper (lower) solutions of (1). For a treatment of upper and lower solutions and the related concepts of subfunctions and superfunctions in the scalar case, see Jackson [8]. For work on vector differential inequalities in addition to [1] mentioned above, see Schmitt [9] and Bebernes and Schmitt [2].

Proof of Theorem 2. Let $D=\left\{(t, X): t \in I,\left|c^{*} X c\right|<c^{*}(|A|+|B|+E) c\right.$ for all $c \in R^{n}$ such that $\left.c^{*} c=1\right\}$. Let $g(t, s)$ be the Green's function associated with $x^{\prime \prime}(t)=0, x(a)=x(b)=0$. For $p$ a positive integer, let $F_{p}(t, X)=F(t, X)+$ $p^{-1}\left(X+X^{*}\right)$, and consider

$$
X^{\prime \prime}=\lambda F_{p}(t, X) \text {. }
$$

Now, $X$ is a solution to (1)-(2), ( $\left.3_{\lambda}\right)-(2)$ and (4 $\left.4_{\lambda}\right)-(2)$, respectively, for $G=F, \lambda F$ and $\lambda F_{p}$ if and only if

$$
X(t)=\int_{a}^{b} g(t, s) G(s, X(s)) d s+Q(t)
$$


It is clear that any such solution is symmetric. The claim is that $(\mathrm{H})$ holds for (4) -(2). That is, if $c^{*} X(t) c=c^{*}(|A|+|B|+E) c$ for some $t \in I$, then there must be a $\tau$ such that $c^{*} X(\tau) c>c^{*}(|A|+|B|+E) c$. Suppose there is a $c \in R^{n}$ and $t_{0} \in I$ such that

$$
c^{*} X\left(t_{0}\right) c=c^{*}(|A|+|B|+E) c
$$

or

$$
c^{*} X\left(t_{0}\right) c=-c^{*}(|A|+|B|+E) c .
$$

Suppose, by way of contradiction, $\left|c^{*} X(t) c\right|<c^{*}(|A|+|B|+E) c$ for all $t \in I$, where $X(t)$ is a solution of $\left(4_{\lambda}\right)-(2)$. To be specific, assume (5) holds, and note that (6) can be handled in a similar manner. Note, the right-hand side of (5) must be positive. It may be assumed that $\lambda>0$, since for $\lambda=0$ the solution must be $Q(t)$ and (5) is false. Define $x(t)=c^{*} X(t) c$. Then $x\left(t_{0}\right)=c^{*}(|A|+|B|+E) c$, is an absolute maximum for $x(t)$. Therefore, $x^{\prime}\left(t_{0}\right)=0$ and $x^{\prime \prime}\left(t_{0}\right)<0$. However,

$$
\begin{aligned}
x^{\prime \prime}\left(t_{0}\right) & =c^{*} \lambda\left[F\left(t_{0}, X\left(t_{0}\right)\right)+p^{-1}\left(X\left(t_{0}\right)+X\left(t_{0}\right)^{*}\right)\right] c \\
& =\lambda c^{*} F\left(t_{0}, X\left(t_{0}\right)\right) c+\lambda p^{-1} c^{*}\left(X\left(t_{0}\right)+X\left(t_{0}\right)^{*}\right) c \\
& \geqslant 0+2 \lambda p^{-1} c^{*} E c=2 \lambda p^{-1}>0 .
\end{aligned}
$$

The $\lambda c^{*} F\left(t_{0}, X\left(t_{0}\right)\right) c$ term is nonnegative since by (5) and the symmetry of $X\left(t_{0}\right)$, there is a positive eigenvalue for $X\left(t_{0}\right)$ making $F\left(t, X\left(t_{0}\right)\right)>0$. This contradiction shows that $(H)$ holds for $\left(4_{\lambda}\right)-(2)$ for each $p \in Z^{+}$.

Thus, by Theorem 1 there is for each $p \in Z^{+}$a solution $X_{p}$ to $\left(4_{1}\right)-(2)$ such that for each $t \in I,\left(t, X_{p}(t)\right) \in D$. By Hartman [8, p. 14] there is a subsequence $X_{p(k)}(t)$ converging to a solution $X(t)$ of (1)-(2) with $(t, X(t)) \in \bar{D}$ for all $t \in I$.

If $A, B>0$ and $c^{*} X(t) c<0$ for some $c \in R^{n}$ and $t \in I$, then for some $t_{0} \in(a, b), x\left(t_{0}\right)<0$ and $x^{\prime}\left(t_{0}\right)=0$. By $A, B>0$ one has $x(a)>0$ and $x(b)>0$. Thus, using the hypothesis on $F$, there exists a $t_{1} \in\left(t_{0}, b\right)$ such that $x(t)<0$ on $\left[t_{0}, t_{1}\right), x\left(t_{1}\right)=0$, and $x^{\prime \prime}(t)<0$ on $\left[t_{0}, t_{1}\right]$. Thus

$$
0 \geqslant \int_{t_{0}}^{t} x^{\prime \prime}(s) d s=x^{\prime}(t)-x^{\prime}\left(t_{0}\right)=x^{\prime}(t)
$$

This implies that

$$
0 \geqslant \int_{t_{0}}^{t_{1}} x^{\prime}(s) d s=x\left(t_{1}\right)-x\left(t_{0}\right)>0,
$$

a contradiction! Hence, the assumption that $c^{*} X(t) c<0$ is false, and it follows that $X(t) \geqslant 0$ for all $t \in I$. A similar argument may be followed to show that $X(t) \leqslant 0$ if $A, B<0$.

Next bounds are put on the matrix $F$, and $F$ will be assumed dependent on $X^{\prime}$ as well as $t$ and $X$ for the following result.

THEOREM 3. Let $A>0, B>0$ and $K=(\sqrt{A}+\sqrt{B})(b-a)^{-1}$. If $0<$ $F\left(t, x, x^{\prime}\right)<2 K^{2}$, then the boundary value problem $\left(1^{\prime}\right)-(2)$ has a positive definite solution on $[a, b]$. 
Proof. Let

$$
\begin{aligned}
& D^{\prime}=\left\{\left(t, X, X^{\prime}\right): t \in I, 0<c^{*} X c<c^{*}(A+B) c,\right. \\
& -(b-a)^{-1} c^{*}(2 A+\sqrt{A} \sqrt{B}+\sqrt{B} \sqrt{A}) c<c^{*} X^{\prime} c<(b-a)^{-1} \\
& \left.\cdot c^{*}(2 B+\sqrt{A} \sqrt{B}+\sqrt{B} \sqrt{A}) c \text { for all } c \in R^{n} \text { with } c^{*} c=1\right\} .
\end{aligned}
$$

As in the proof of Theorem 2, it is clear that any solution is symmetric. Further if the solution stays in $D^{\prime}$ it will in fact be positive definite. Next it is shown that $\left(\mathrm{H}^{\prime}\right)$ holds. If $\left(\mathrm{H}^{\prime}\right)$ does not hold, one of the above four matrix inequalities fails. This cannot happen when $\lambda=0$, since then $Q(t)$ is the unique solution. Consider $0<c^{*} X c$. If there is $t_{0} \in(a, b)$ and $c^{*} c=1$ such that $0=c^{*} X\left(t_{0}\right) c$ and $c^{*} X(t) c$ $\geqslant 0$ for all $t \in[a, b]$, then for $x(t)=c^{*} X(t) c$ one has that $x\left(t_{0}\right)=x^{\prime}\left(t_{0}\right)=0$ and $0<x^{\prime \prime}(t)<2 \lambda c^{*} K^{2} c<2 c^{*} K^{2} c$. Integrating this inequality twice on each side of $t_{0}$

$$
x(b)<\left(b-t_{0}\right)^{2} c^{*} K^{2} c \text { and } x(a)<\left(t_{0}-a\right)^{2} c^{*} K^{2} c
$$

are obtained. Taking square roots of these inequalities and adding them leads to

$$
\sqrt{c^{*} A c}+\sqrt{c^{*} B c}<\sqrt{c^{*}(\sqrt{A}+\sqrt{B})^{2} c} .
$$

Squaring this inequality and simplifying one obtains

$$
2 \sqrt{c^{*} A c \cdot c^{*} B c}<c^{*}(\sqrt{A} \sqrt{B}+\sqrt{B} \sqrt{A}) c .
$$

But this contradicts the Cauchy-Schwarz inequality, which in this notation is

$$
c^{*} \sqrt{A} \sqrt{B} c \leqslant \sqrt{c^{*} \sqrt{A} \sqrt{A} c \cdot c^{*} \sqrt{B} \sqrt{B} c} \text {. }
$$

Now if $\left(\mathrm{H}^{\prime}\right)$ fails for $c^{*} X c<c^{*}(A+B) c$, there will be $t_{0} \in(a, b)$ and $c^{*} c=1$ such that

$$
c^{*} X\left(t_{0}\right) c=c^{*}(A+B) c \text { and } c^{*} X(t) c<c^{*}(A+B) c
$$

for all $t \in I$. Then

$$
x^{\prime}\left(t_{0}\right)=0 \text { and } x^{\prime \prime}\left(t_{0}\right)=c^{*} F\left(t_{0}, X\left(t_{0}\right), X^{\prime}\left(t_{0}\right)\right) c>0 .
$$

Hence, there is a strict relative minimum for $x(t)$ at $t_{0}$. Thus, $x(\tau)>c^{*}(A+B) c$ for some $\tau$ near $t_{0}$, and $\left(\mathrm{H}^{\prime}\right)$ does not fail. Going to the last inequality note that $\left(\mathrm{H}^{\prime}\right)$ cannot fail on $[a, b)$ since $c^{*} X^{\prime \prime}(t) c>0$. Thus $b$ is the only value left to check. Suppose $\left(\mathrm{H}^{\prime}\right)$ fails for the last inequality. Then there is $c^{*} c=1$ such that

$$
c^{*} X^{\prime}(b) c=(b-a)^{-1} c^{*}(2 B+\sqrt{A} \sqrt{B}+\sqrt{B} \sqrt{A}) c .
$$

Letting $x(t)=c^{*} X(t) c$ and integrating the inequality

$$
x^{\prime \prime}(t)<2(b-a)^{-2} c^{*}(\sqrt{B}+\sqrt{A}) c
$$

twice, the contradictory inequality $c^{*}(\sqrt{B}+\sqrt{A}) c<c^{*}(\sqrt{B}+\sqrt{A}) c$ is obtained. A similar argument shows that $\left(H^{\prime}\right)$ holds with respect to the third inequality with the roles of the endpoints reversed.

A question arises from this study, as might be inferred by the remark following Theorem 2, of whether sufficient conditions for existence of solutions to the boundary value problem might be given in terms of upper and lower solutions. For 
example, such a condition might be that, for $A, B$ symmetric matrices, there exists an upper solution through $(a, C),(b, D)$ for each $C>A$ and $D>B$, and there exists a lower solution through $(a, C),(b, D)$ for each $C<A$ and $D<B$. The author knows of no work in this direction.

A difference between Theorem 1 above and Theorem 2.1 of [1] is the seemingly unnatural $(1-\lambda) x$ term on the right-hand side of equation (4) in [1]. This term is necessary in [1] since the boundary value problem

$$
\begin{gathered}
x^{\prime \prime}=0, \\
x(0)=x(1), \quad x^{\prime}(0)=x^{\prime}(1)
\end{gathered}
$$

does not have unique solutions. But if (7) is replaced by

$$
x^{\prime \prime}-x=0,
$$

then the resulting boundary value problem (9)-(8) generates unique solutions. In Theorem 1, above, the corresponding boundary value problem is the matrix version of (7) together with $X(a)=X(b)=0$ where uniqueness is not a problem.

\section{REFERENCES}

1. J. W. Bebernes, $A$ simple alternative problem for finding periodic solutions of second order ordinary differential systems, Proc. Amer. Math. Soc. 42 (1974), 121-127.

2. J. W. Bebernes and K. Schmitt, Periodic boundary value problems for systems of second order differential equations, J. Differential Equations 13 (1973), 32-47.

3. R. Bellman, Introduction to matrix analysis, McGraw-Hill, New York, 1970.

4. L. Fountain and L. Jackson, $A$ generalized solution of the boundary value problem for $y^{\prime \prime}=$ $f\left(x, y, y^{\prime}\right)$, Pacific J. Math. 12 (1962), 1251-1272.

5. P. Hartman, Ordinary differential equations, Wiley, New York, 1964.

6. K. A. Heimes, Two point boundary problems in Banach space, J. Differential Equations 5 (1969), 215-225.

7. __ Green's functions for linear second order systems, SIAM J. Math. Anal. 9 (1978), 207-214.

8. L. K. Jackson, Subfunctions and second-order ordinary differential inequalities, Adv. in Math. 2 (1968), 307-363.

9. K. Schmitt, Periodic solutions of systems of second-order differential equations, J. Differential Equations 11 (1972), 180-192.

10. J. Schwartz, Nonlinear functional analysis, McGraw-Hill, New York, 1970.

11. E. C. Tomastik, Oscillation of nonlinear matrix differential equations of the second order, Proc. Amer. Math. Soc. 19 (1968), 1427-1431.

Department of Mathematical Sciences, North Dakota State University, fargo, North DAKota 58105 